\title{
Seismic shakedown design of frames based on a probabilistic approach
}

\author{
S. Benfratello, L. Palizzolo \& P. Tabbuso \\ Department of Civil, Environmental, Aerospace, Material Engineering, \\ University of Palermo, Italy
}

\begin{abstract}
The present study concerns the optimal design of elastic perfectly plastic structures subjected to a combination of fixed and seismic loads. In particular, plane frames are considered and suitable measures of the beam element cross sections are chosen as design variables. The optimal design is required to behave in a purely elastic manner when subjected just to the fixed load and to have the capability to eventually shakedown when simultaneously subjected to fixed and seismic loads. Due to the natural uncertainness related to the definition of the seismic load history, a new probabilistic approach is proposed, consisting into two subsequent search steps. At first a suitably chosen large number of minimum volume designs are obtained for as many random seismic load histories deduced by a suitably chosen reference power spectral density function, determining a probabilistic distribution of optimal volumes. Subsequently, the volume obtained with probability 1 is assigned as the optimal structural volume, and a new optimal design problem is solved in order to obtain the material optimal distribution. The latter is a minimum elastic strain energy one for fixed volume. The performed applications confirm the effectiveness of the proposed procedure. Keywords: minimum volume design, seismic loading, shakedown behaviour, probabilistic approach.
\end{abstract}

\section{Introduction}

Structural optimization is a topic of fundamental interest in structural engineering (see, e.g. [1-8]). As it is well known, the formulation of a structural optimization problem requires, at first, the definition of a suitable objective function involving appropriate relevant structural parameters (design variables). 
The latter can concern the geometry, the elastic properties, the topology of the structure, etc. Some admissible ranges for the design variables are usually adopted and the state equations are introduced. Furthermore, appropriate admissibility criteria are defined. These criteria are usually written in terms of behavioural constraints for the structure, very often they express mechanical conditions and they usually identify with different structural limit conditions.

The structural optimization problems are very often formulated as search for the minimum structural weight, which substantially provides a quantity proportional with the minimum cost to be suffered for the structure construction.

On the other side, the choice of the admissibility conditions is usually complex and specific of the particular optimization problem to be formulated. These conditions are represented by inequalities identifying one or more limit behaviour for the material or the structure. If reference is made to elastic plastic structures, the limit conditions can characterize: the purely elastic behaviour; the elastic shakedown behaviour; the plastic shakedown behaviour and/or the incremental collapse; the instantaneous collapse (see, e.g. [9-13]). An important aspect to be faced is that the most dangerous loads (wind, earthquake) are timedependent and random. Most of the effected study are related to a deterministic approach (see, e.g. [14-16]), even if some paper exists accounting for the randomness of the load, but limiting to the structure elastic behaviour (see, e.g. $[17,19])$. As a consequence, an efficient and general optimization tool which takes into account even these two characteristics is needed.

The aim of the present paper is to propose a new probabilistic approach for continuous variable optimal structural design of steel frames taking into account the randomness of the loading. The optimality condition is focused on the elastic response under fixed loads and on the shakedown behaviour under a combination of fixed and seismic loads. The dynamic structural response is obtained in the time domain by integrating the equation of motion by means of the suitable tools available in literature. The proposed approach can be synthetically described as constituted by the following steps:

1) an appropriate large number of accelerograms are generated from an assigned power spectral density function by means of the Monte Carlo approach;

2) for each accelerogram, a minimum volume design problem for the elastic perfectly plastic frame structure subjected to the assigned combination of fixed loads and seismic actions is solved;

3 ) the obtained large number of volumes is random as the related generated accelerogram on which they depend and they are used to define the corresponding volume cumulative probability function;

4) the volume with probability 1 is assigned as optimal value $\left(V_{\text {opt }}\right)$;

5) a load domain is defined by the accelerograms generated at the step 1) whose corresponding volume is not greater than a suitable per cent of $V_{\text {opt }}$;

6) the final optimal design is obtained as the optimal distribution of material which minimizes the corresponding strain energy function, being assigned the optimal volume defined at step 4), for the structure subjected to any load history within the dynamic load domain introduced at step 5). 
In order to build the referenced seismic load domain a suitable number of accelerograms are chosen defining the relevant basic conditions. The considered accelerograms are those related to a suitably chosen interval of the volume cumulative distribution function where the upper bound is the selected per cent of the optimal volume. Once defined the relevant dynamic load domain, any admissible dynamic load history is given as a suitable combination of the basic load conditions, following the unrestricted dynamic shakedown theory $[20,21]$. A numerical application is effected, related to a flexural two-floor two-span elastic perfectly plastic plane steel frame.

\section{The frame structure and the load modelling}

In this section some fundamentals, mainly regarding the definition of appropriate model both for the frame structure and for the acting loads, are introduced.

As it has been stated in the introduction section the structure typology examined in this paper is a frame one. The frame is constituted by beam elements described by the Navier kinematical model (Fig. 1a), b)).

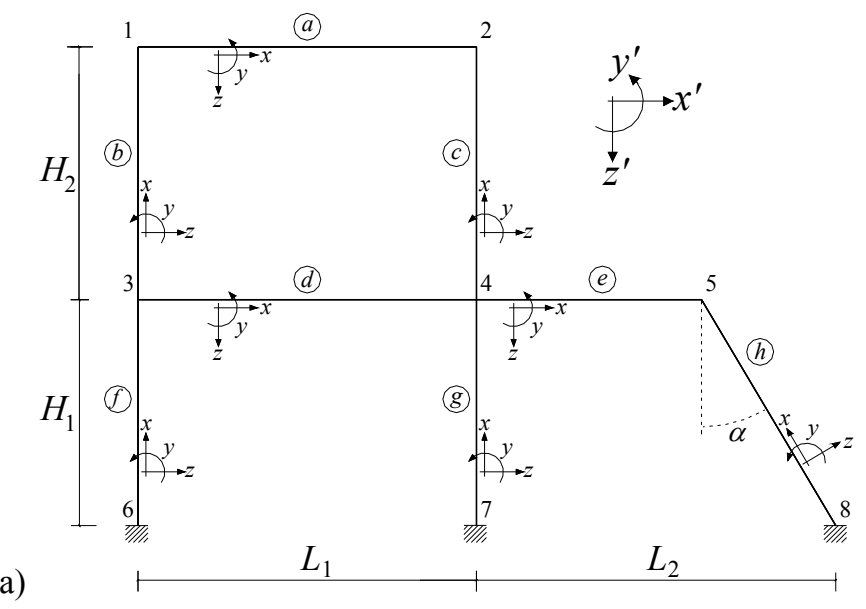

a)

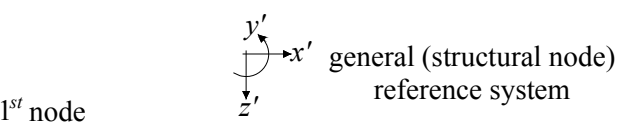

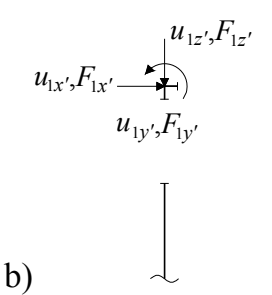

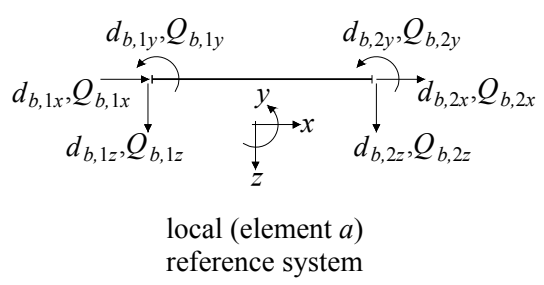

reference system $2^{n d}$ node

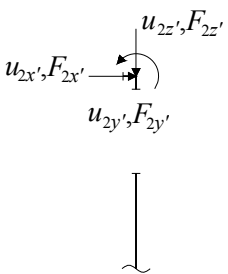

Figure 1: a) Plane frame; b) mechanical and kinematical quantities. 
Since the material element behaviour beyond the elastic limit has to be considered, appropriate plastic hinges are located at the extremes of each element and in the middle points, where necessary (Fig. 2).

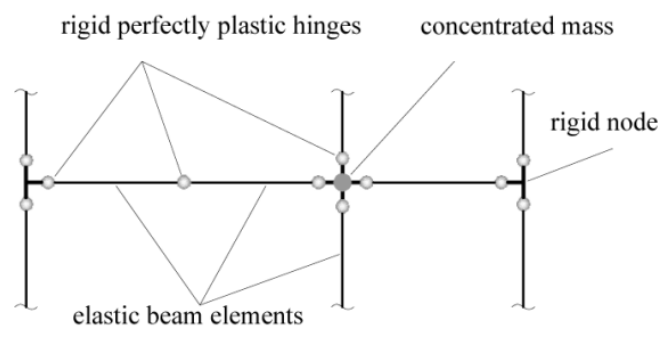

Figure 2: Location of the plastic hinges.

The loadings acting on the frame structure are of two different types: the first one is the fixed load related to the structural weight and to the relevant permanent actions; the second one is related to the seismic actions and it is intrinsically random in intensity as well as dynamic; the two described loads, simultaneously acting, determine the reference load combination. The two emphasized seismic loading features represent two fundamental aspects to be faced by the structural designer. Furthermore, it is worth noticing that a structure can suffer several seismic loadings during its life, each different for intensity and duration. In this paper attention is focused on the shakedown behaviour of the structure and, as a consequence, it is necessary to refer to the theory of shakedown under dynamic loadings.

In literature this problem can be faced in the framework of the Ceradini theorem [22]; however this theorem can be applied when the acting load on the structure is regarded as a never-ending one. The latter assumption is unrealistic especially when a seismic action is considered. Another approach is that of the so-called unrestricted dynamic shakedown $[20,21]$ which requires the definition of an appropriate domain characterized by some known basic load conditions. Clearly, this approach conflicts with the randomness of the seismic action. In order to account for the described features in the paper the probabilistic approach described at the end of the foregoing section is proposed.

Making reference to the seismic actions, let us consider the relevant frame as a flexural plane frame characterized by $n_{b}$ beam-type elements, $n_{N}$ standard nodes (each characterized by three degrees of freedom), by masses concentrated at each node and just subjected to an horizontal ground acceleration $\ddot{u}_{g}(t)$. The structure is modelled as a Multi-Degree-Of-Freedom (MDOF) one and the dynamic equilibrium equations can be written in the following form:

$$
\boldsymbol{M} \ddot{\boldsymbol{u}}(t)+\boldsymbol{B} \dot{\boldsymbol{u}}(t)+\boldsymbol{K} \boldsymbol{u}(t)=\boldsymbol{f}_{g}(t),
$$


being $\boldsymbol{u}(t), \dot{\boldsymbol{u}}(t)$ and $\ddot{\boldsymbol{u}}(t)$ the nodal displacement, velocity and acceleration vectors, respectively, where the over dot means time derivative; furthermore, $\boldsymbol{f}_{g}(t)=-\boldsymbol{M} \boldsymbol{\tau} \ddot{u}_{g}(t)$ is the loading vector, $\boldsymbol{M}$ and $\boldsymbol{B}$ are the mass and damping matrices of order $3 \cdot n_{N}, \boldsymbol{K}=\tilde{\boldsymbol{C}} \boldsymbol{D C}$ is the frame external square stiffness matrix of order $3 \cdot n_{N}$ and $\boldsymbol{\tau}$ is the $3 \cdot n_{N}$ influence vector. $\boldsymbol{C}$ is the compatibility matrix of dimensions $6 \cdot n_{b} \times 3 \cdot n_{N}$ containing suitable defined coordinate transformation matrices related to each beam element (being its transpose $\tilde{\boldsymbol{C}}$ the equilibrium matrix) and $\boldsymbol{D}$ is the frame internal (square block diagonal) stiffness matrix of order $6 \cdot n_{b}$. The initial conditions associated to eqn (1) are assumed to be $\boldsymbol{u}(0)=\mathbf{0}, \dot{\boldsymbol{u}}(0)=\mathbf{0}$. Eqn (1) can be solved directly by making reference to the tools available in literature [23] or with a classical modal technique. Once $\boldsymbol{u}(t)$ is known for each instant, it is possible to calculate the beam extreme cross section displacement vector and the generalized stress one by means of the following formulas:

$$
\begin{gathered}
\boldsymbol{d}(t)=\boldsymbol{C} \boldsymbol{u}(t), \\
\boldsymbol{Q}(t)=\boldsymbol{D} \boldsymbol{d}(t)+\boldsymbol{Q}^{*}(t),
\end{gathered}
$$

where $\boldsymbol{Q}^{*}(t)$ is the perfectly clamped generalized stress vector.

In the following, the interest is focused only to the case of undulatory dynamic effect. As a consequence it is usually to modify eqn (1) by means of the so-called static condensation [23].

As usual in seismic engineering a ground motion sample $\ddot{u}_{g}$ can be generated as a superposition of $n$ single-frequency wave components of the spectrum $\omega_{i}$ :

$$
\ddot{u}_{g}(t)=E(t) \cdot \sum_{i=1}^{n} \sqrt{2 S\left(\omega_{i}\right) \Delta \omega} \cdot \cos \left(\omega_{i} t+\varphi_{i}\right)
$$

where $E(t)$ is an envelope shape function aimed at modeling the evolutive character of the seismic wave, $S\left(\omega_{i}\right)$ is the one-sided power spectral density function (PSD), $\Delta \omega=\omega_{c} / n$ is the sample frequency (being $\omega_{c}$ the cut-off frequency), and $\varphi_{i}$ is a random phase angle uniformly distributed in $0 \div 2 \pi$. In particular, as shape function, the Iwan-Hou model is here adopted:

$$
E(t)=\left[\left(\frac{\beta}{m}\right)^{m} \exp (m)\right] t^{m} \exp (-\beta t)
$$


where $m$ and $\beta$ are suitable positive parameters, while as PSD it's used the well-known Kanai-Tajimi filter model:

$$
S(\omega)=\frac{\left(\omega_{g}^{4}+4 \zeta_{g}^{2} \omega_{g}^{2} \omega^{2}\right) S_{W}}{\left(\omega_{g}^{2}-\omega^{2}\right)+4 \zeta_{g}^{2} \omega_{g}^{2} \omega^{2}}
$$

where $\omega_{g}, \zeta_{g}$ are parameters related to the site soil characteristics and $S_{W}$ is the power spectral density of the white noise process.

\section{Proposed optimal design problem formulation}

Let us consider an elastic perfectly plastic frame structure as above described and, according to the assumed loading model, let it be subjected to fixed mechanical loads and to seismic loads. The optimal design problem that we want to formulate is a minimum volume one, so that as design variables some appropriate measures of the beam element cross sections are chosen. Let the typical $v^{\text {th }}$ beam element cross section geometry be fully described by the $r$ components of the vector $\boldsymbol{h}_{v}\left(v=1,2, \ldots, n_{b}\right)$ so that $\tilde{\boldsymbol{h}}=\left[\tilde{\boldsymbol{h}}_{1}, \tilde{\boldsymbol{h}}_{2}, \ldots, \tilde{\boldsymbol{h}}_{v}, \ldots, \tilde{\boldsymbol{h}}_{n_{b}}\right]$ represents the $n_{b} \times r$ supervector collecting all the design variables. The structure is required to remain purely elastic when no seismic actions occur and to exhibit an elastic shakedown behaviour for the combination of fixed and seismic loads. For the typical $k^{\text {th }}$ seismic load history the minimum volume design problem formulation can be written as follows:

$$
\min _{\left(\boldsymbol{h}, \boldsymbol{u}_{0}, \boldsymbol{u}_{k}, \boldsymbol{\rho}\right)} V_{k}
$$

subjected to:

$$
\begin{gathered}
\boldsymbol{h}_{\min } \leq \boldsymbol{h} \leq \boldsymbol{h}_{\max }, \\
\boldsymbol{\tau} \boldsymbol{h}-\overline{\boldsymbol{h}} \geq \mathbf{0}, \\
\boldsymbol{K} \boldsymbol{u}_{0}=\boldsymbol{F}_{0}^{*}, \\
\boldsymbol{Q}_{0}=\boldsymbol{D} \boldsymbol{C} \boldsymbol{u}_{0}+\boldsymbol{Q}_{0}^{*}, \\
\boldsymbol{M} \ddot{\boldsymbol{u}}_{k}(t)+\boldsymbol{B} \dot{\boldsymbol{u}}_{k}(t)+\boldsymbol{K} \boldsymbol{u}_{k}(t)=-\boldsymbol{M} \boldsymbol{\tau} \ddot{u}_{g k}(t), \\
\boldsymbol{Q}_{s k}(t)=\boldsymbol{D} \boldsymbol{C} \boldsymbol{u}_{k}(t), \\
\boldsymbol{P}_{0}=\tilde{\boldsymbol{N}} \tilde{\boldsymbol{G}}_{p} \boldsymbol{Q}_{0}, \\
\boldsymbol{P}_{s k}=\max _{0 \leq t \leq T} \tilde{\boldsymbol{N}}_{p} \boldsymbol{Q}_{s k}(t), \\
\boldsymbol{\varphi}^{E}=\boldsymbol{P}_{0}-\boldsymbol{R} \leq \mathbf{0},
\end{gathered}
$$




$$
\begin{gathered}
\boldsymbol{\varphi}_{k}^{S}=\boldsymbol{P}_{0}+\boldsymbol{P}_{s k}+\boldsymbol{\rho}-\boldsymbol{R} \leq \mathbf{0}, \\
\tilde{\boldsymbol{A}} \boldsymbol{\rho}=\mathbf{0} .
\end{gathered}
$$

In eqns $(7 b, c) \boldsymbol{h}$ is the design variable vector, $\boldsymbol{\tau}$ is the technological constraint matrix and $\overline{\boldsymbol{h}}$ represents a suitably chosen technological vector. In eqns ( $7 \mathrm{~d}-\mathrm{g}) \boldsymbol{u}_{0}$ and $\boldsymbol{Q}_{0}$ are the purely elastic response to the assigned full fixed loads in terms of frame node displacements and generalized stresses evaluated at the extremes of the beam elements, respectively, while $\boldsymbol{u}_{k}(t)$ and $\boldsymbol{Q}_{s k}(t)$ are the analogous purely elastic response but to the $k^{\text {th }}$ seismic load history. In eqns $\left(7 \mathrm{~h}\right.$, i) $\boldsymbol{P}_{0}$ is the required plastic capacity vector related to the fixed loads and $\boldsymbol{P}_{s k}$ is the analogous required plastic capacity but related to the $k^{\text {th }}$ seismic load history, being $\tilde{N}$ the matrix of the unit external normals to the elastic domain whose boundary is assumed as constituted by a discrete number of sides and $\tilde{\boldsymbol{G}}_{p}$ an appropriate equilibrium matrix which applied to element nodal generalized stresses provides the generalized stresses acting upon the plastic nodes of the elements. Furthermore, in eqns $(7 \mathrm{j}-\ell) \boldsymbol{\varphi}^{E}$ and $\boldsymbol{\varphi}_{k}^{S}$ are the plastic potential vectors related to the purely elastic limit (apex $E$ ) and to the elastic shakedown limit (apex $S$ ), respectively, $\rho$ is the self-equilibrated stress vector satisfying the

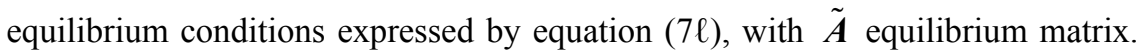
Finally, $\boldsymbol{R}$ is the relevant plastic resistance vector.

According to the approach previously described, the optimal search problem (7) has to be solved for each generated time history of ground acceleration. Subsequently, the cumulative distribution function of the obtained volumes is obtained and the value with probability 1 is assigned as corresponding optimal value $V_{\text {opt }}$ for the volume. Once deduced this optimal value, the optimal structure design can be founded as the one of maximum volume $V_{\text {opt }}$ and such that the material distribution ensures the minimum of the strain energy. In order to formulate this latter problem, according to the unrestricted shakedown theory, the structure is thought as subjected to a combination of the assigned fixed loads and of any seismic load history within a suitably defined seismic load domain. The seismic load domain is characterized by a finite number $(\mathrm{m})$ of assigned dynamic basic load histories identified as the ones related to volume values appertaining to a suitably chosen interval of the volume cumulative probability function where the upper bound is a selected per cent of the optimal volume. Therefore the minimum strain energy optimal design can be formulated as follows:

$$
\min _{\left(h, u_{0}, \boldsymbol{u}_{i}, \boldsymbol{\rho}\right)} \sum_{i=1}^{m} \int_{0}^{T} \tilde{\hat{\boldsymbol{Q}}}_{i}(t) \hat{\boldsymbol{\Phi}} \hat{\boldsymbol{Q}}_{i}(t) d t
$$


subjected to:

$$
\begin{gathered}
V \leq V_{\mathrm{opt}}, \\
\boldsymbol{h}_{\min } \leq \boldsymbol{h} \leq \boldsymbol{h}_{\max }, \\
\tau \boldsymbol{\boldsymbol { h }}-\overline{\boldsymbol{h}} \geq \mathbf{0}, \\
\boldsymbol{K} \boldsymbol{u}_{0}=\boldsymbol{F}_{0}^{*}, \\
\boldsymbol{Q}_{0}=\boldsymbol{D} \boldsymbol{C} \boldsymbol{u}_{0}+\boldsymbol{Q}_{0}^{*}, \\
\boldsymbol{M} \ddot{\boldsymbol{u}}_{i}(t)+\boldsymbol{B} \dot{\boldsymbol{u}}_{i}(t)+\boldsymbol{K} \boldsymbol{u}_{i}(t)=-\boldsymbol{M} \boldsymbol{\tau} \ddot{u}_{g i}(t), i=1,2, \ldots, m \\
\boldsymbol{Q}_{s i}(t)=\boldsymbol{D} \boldsymbol{C} \boldsymbol{u}_{i}(t), i=1,2, \ldots, m \\
\hat{\boldsymbol{Q}}_{0}=\tilde{\boldsymbol{G}}_{p} \boldsymbol{Q}_{0}, \quad i=1,2, \ldots, m \\
\hat{\boldsymbol{Q}}_{s i}(t)=\tilde{\boldsymbol{G}}_{p} \boldsymbol{Q}_{s i}(t), i=1,2, \ldots, m \\
\hat{\boldsymbol{Q}}_{i}(t)=\hat{\boldsymbol{Q}}_{0}+\hat{\boldsymbol{Q}}_{s i}(t), \quad i=1,2, \ldots, m \\
\boldsymbol{P}_{0}=\tilde{\boldsymbol{N}} \hat{\boldsymbol{Q}}_{0}, \\
\boldsymbol{P}_{s i}=\max _{0 \leq t \leq T} \tilde{\boldsymbol{N}} \hat{\boldsymbol{Q}}_{s i}(t), i=1,2, \ldots, m \\
\boldsymbol{\varphi}^{E}=\boldsymbol{P}_{0}-\boldsymbol{R} \leq \mathbf{0}, \\
\boldsymbol{\varphi}_{i}^{S}=\boldsymbol{P}_{0}+\boldsymbol{P}_{s i}+\boldsymbol{\rho}-\boldsymbol{R} \leq \mathbf{0}, i=1,2, \ldots, m \\
\tilde{\boldsymbol{A}} \boldsymbol{\rho}=\mathbf{0} .
\end{gathered}
$$

In problem (8), besides the already known symbols, $\hat{\boldsymbol{Q}}_{0}$ are the generalized stresses evaluated at the plastic nodes of the elements related to the fixed loads, $\hat{\boldsymbol{Q}}_{s i}(t)$ are the generalized stresses evaluated at the plastic nodes of the elements related to the $i^{\text {th }}$ seismic basic load condition and $\hat{\boldsymbol{Q}}_{i}(t)$ are the total generalized stresses evaluated at the plastic nodes of the elements related to the combination of fixed loads and $i^{\text {th }}$ seismic basic load condition. The total strain energy is evaluated in correspondence of the plastic nodes of the elements being $\hat{\boldsymbol{\Phi}}$ the relevant flexibility matrix.

\section{Applications}

The studied frame is constituted by a two floors and two spans structure as plotted in Fig. 3a). All the beam elements have square box cross section with edge $a=200 \mathrm{~mm}$ and constant thickness $h$ variable between $h_{\min }=4 \mathrm{~mm}$ and $h_{\max }=40 \mathrm{~mm}$ (Fig. 3b)). Furthermore, $L_{1}=600 \mathrm{~cm}, L_{2}=400 \mathrm{~cm}, H=500 \mathrm{~cm}$, Young modulus $E=21 \mathrm{MN} / \mathrm{cm}^{2}$ and yield stress $\sigma_{y}=23.5 \mathrm{kN} / \mathrm{cm}^{2}$ have been assumed. 


\section{a)}

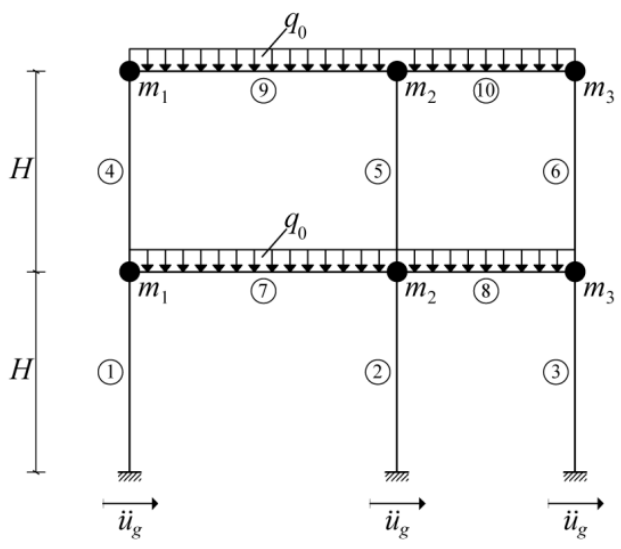

)

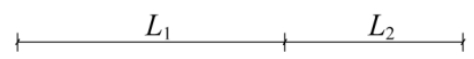

b)

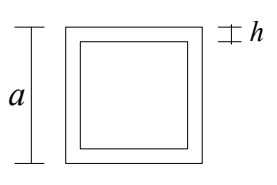

Figure 3: The plane frame structure: a) geometry and load condition; b) square box cross sections of the elements.

Two rigid perfectly plastic hinges are located at the extremes of all the elements, considered to be purely elastic, and an additional hinge is located in the middle point of the longer beams. The interaction between bending moment $M$ and axial force $N$ has been taken into account. In Fig. 4 the dimensionless rigid plastic domain of the typical plastic hinge is plotted in the plane $\left(N / N_{y}\right.$, $M / M_{y}$ ), being $N_{y}$ and $M_{y}$ the yield generalized stresses corresponding to $N$ and $M$, respectively.

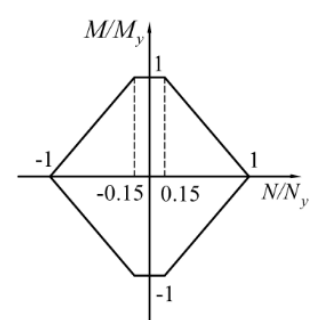

Figure 4: Dimensionless rigid plastic domain of the typical plastic hinge.

The structure beams are all subjected to the same fixed uniformly distributed vertical load, $q_{0}=30 \mathrm{kN} / \mathrm{m}$. Referring to the dynamic loads, we assume that the seismic masses are located at all the structure nodes; due to the described gravitational load $q_{0}$ they are equal at each floor and it results: $m_{1}=7.34 \mathrm{kN} \cdot \mathrm{s}^{2} / \mathrm{m}, \quad m_{2}=12.23 \mathrm{kN} \cdot \mathrm{s}^{2} / \mathrm{m}, \quad m_{3}=4.90 \mathrm{kN} \cdot \mathrm{s}^{2} / \mathrm{m}$. The values 
assigned to the seismic masses are computed considering that during the earthquake the gravitational loads do not act all simultaneously on the structure, so that it is possible to evaluate $m_{i}=\left(0.8 \cdot q_{0} \cdot \bar{L}_{i}\right) / g$, with $g$ the acceleration of gravity, $i=1,2,3$ and $\bar{L}_{i}$ relevant influence beam length. At the same manner, the generalized stresses to combine with the seismic response are deduced by a suitably reduction of $q_{0}$.

The solution to problem (7) has been obtained for $k=1,2, \ldots, 1000$ utilizing a harmony search algorithm and the related obtained volumes allowed us to identify the relevant volume cumulative distribution function, plotted in Fig. 5.

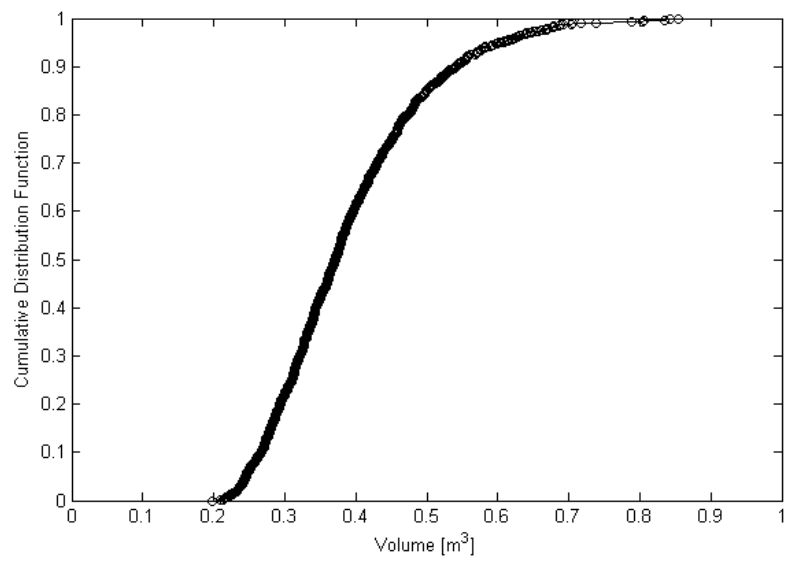

Figure 5: Volume cumulative distribution function of the plane frame volumes.

The volume with probability 1 is $V_{\text {opt }}=0.855 \mathrm{~m}^{3}$. For this optimal volume the thickness distribution reported in Table 1 is associated.

Table 1: Thicknesses related to the optimal volume $V_{\text {opt }}=0.855 \mathrm{~m}^{3}$.

\begin{tabular}{lllllllllll}
\hline El. & $\mathbf{1}$ & $\mathbf{2}$ & $\mathbf{3}$ & $\mathbf{4}$ & $\mathbf{5}$ & $\mathbf{6}$ & $\mathbf{7}$ & $\mathbf{8}$ & $\mathbf{9}$ & $\mathbf{1 0}$ \\
\hline$h$ & 28.9 & 39.5 & 33.9 & 10.1 & 25.6 & 14.0 & 27.1 & 4.0 & 17.8 & 14.8 \\
\hline
\end{tabular}

In order to perform the second optimization problem (8) a suitable seismic load domain has been defined. In particular, five seismic acceleration histories have been considered as basic conditions: the one related to the value $0.95 \cdot V_{\text {opt }}$ and the immediately preceding four related to volume values not greater than $0.95 \cdot V_{\mathrm{opt}}$. The minimum strain energy optimal shakedown design problem, according to the unrestricted dynamic shakedown approach, solved by means of a suitable harmony search algorithm, provides a thickness distribution as reported in Table 2. 
Table 2: Thicknesses related to the final optimal design.

\begin{tabular}{lllllllllll}
\hline El. & $\mathbf{1}$ & $\mathbf{2}$ & $\mathbf{3}$ & $\mathbf{4}$ & $\mathbf{5}$ & $\mathbf{6}$ & $\mathbf{7}$ & $\mathbf{8}$ & $\mathbf{9}$ & $\mathbf{1 0}$ \\
\hline$h$ & 20.7 & 29.4 & 29.3 & 17.3 & 24.7 & 6.9 & 33.6 & 31.2 & 33.2 & 17.8 \\
\hline
\end{tabular}

As it is possible to observe, a consistent redistribution of material is obtained and, in the special examined case, the thickness in the beams strongly increases. On the other side, the required behaviour for the structure is an elastic shakedown one and, as a consequence, a brittle collapse mechanism of the frame is not expected.

\section{Conclusions}

The present paper has been devoted to the proposing of a new probabilistic approach to the optimal design of elastic perfectly plastic structures subjected to a combination of fixed and seismic loads. In particular, reference has been made to steel frames and the optimal design problem has been formulated as the search for the minimum volume of the structure required to purely elastically respond to the fixed loads and capable to eventually shakedown for the combination of fixed and seismic loads. The novelty of the proposal is related to the probabilistic approach which characterizes the procedure. Actually, being random in nature the seismic load, the optimal volume is defined as that value corresponding to the probability 1 deduced by the related volume cumulative distribution function, and the final design is reached solving two subsequent optimal design problems. The first one is a minimum volume one and it is solved for a suitably defined large number of seismic load history generated starting from an assigned power spectral density function by means of the Monte Carlo approach. The second one is a minimum strain energy one for the structure with a volume not greater than the above defined optimal one. Two different computational algorithms have been utilized for the solution of the relevant optimization problems. For both problems a suitable modified harmony search algorithm has been utilized.

\section{References}

[1] Massonet, C.E. \& Save, M., Plastic analysis and design, Blaisdell Publishing Company, New York, 1965.

[2] Gallagher, R.H. \& Zienkiewicz, O.C., Optimum structural design, London: John Wiley \& Sons, 1973.

[3] Rozvany, G.I.N., Optimal design of flexural systems, Oxford: Pergamon Press, 1976.

[4] Save, M.A. \& Prager, W., Structural optimization, New York: Plenum Press, 1985.

[5] Haug, E.J., Choi, K.K. \& Komkov, V., Design Sensitivity Analysis of Structural Systems, Academic Press, New York, 1986.

[6] Brousse, P., Optimization in mechanics: problems and methods, The Nederlands, Amsterdam: Elsevier Science Publishers, 1988. 
[7] Banichuk, N.V., Introduction to optimization of structures, New York: Springer-Verlag, 1990.

[8] Atkočiūnas, J., Optimal shakedown design of elastic-plastic structures, VGTU Publishing House TECHNIKA, Vilnius, 2011.

[9] Cinquini, C., Guerlement, G. \& Lamblin, D., Finite element iterative methods for optimal elastic design of circular plates, Comp. Struct., 12(1), pp. 85-92, 1980.

[10] Giambanco, F., Palizzolo, L. \& Polizzotto, C., Optimal shakedown design of beam structures, Struct. Opt., 8, pp. 156-167, 1994.

[11] Maier, G., Srinivasan, R. \& Save, M., On limit design of frames using linear programming, J. Struct. Mech., 4, pp. 349-378, 1976.

[12] Palizzolo, L., Optimization of continuous elastic perfectly plastic beams, Comp. \& Struct., 82(4-5), pp. 397-411, 2004.

[13] Giambanco, F., Palizzolo, L. \& Caffarelli, A., Computational procedures for plastic shakedown design of structures, Struct. Mult. Opt., 28(5), pp. 317-329, 2004.

[14] Benfratello, S., Giambanco, F., Palizzolo, L. \& Tabbuso, P., Optimal design of steel frames accounting for buckling, Meccanica, 48(9), pp. 2281-2298, 2013.

[15] Benfratello, S., Palizzolo, L. \& Tabbuso, P., Dynamic Shakedown Design of Structures under Repeated Seismic Loads, The Fifth Int. Conf. Struct. Eng., Mech. and Comp., Cape Town - South Africa, 2013.

[16] Benfratello, S., Palizzolo, L. \& Tabbuso, P., Optimal design of steel frames accounting for seismic protection devices, Struct. Mult. Opt., 49(1), pp. 93-106, 2014.

[17] Spence, S.M.J. \& Gioffrè, M., Efficient Algorithms for the Reliability Optimization of Tall Buildings, J. Wind Eng. Ind. Aerod., 99, pp. 691-699, 2011.

[18] Spence, S.M.J. \& Gioffrè, M., Large Scale Reliability-Based Design Optimization of Wind Excited Tall Buildings, Prob. Eng. Mech., 28, pp. 206-215, 2012.

[19] Spence, S.M.J. \& Kareem, A., Data-Enabled Design and Optimization (DEDOpt): Tall Steel Building Frameworks, Comp. Struct., 129(12), pp. 134-147, 2013.

[20] Borino, G., \& Polizzotto, C., Dynamic shakedown of structures under repeated seismic loads. J. Eng. Mech. ASCE, 121(12), pp. 1306-1314, 1995.

[21] Polizzotto, C., Dynamic shakedown of elastic-plastic solids for a set of alternative loading histories. J. Non Linear Mech., 19(4), pp. 363-371, 1984.

[22] Ceradini, G., Dynamic shakedown in elastic-plastic bodies. J Eng. Mech. Div. ASCE, 106(EM3), pp. 481-499, 1980.

[23] Muscolino, G., Dinamica delle strutture con fondamenti ed applicazioni di ingegneria sismica e dinamica aleatoria, Pitagora Editrice, 2012. 